Check for updates

Cite this: Mater. Horiz., 2019, 6, 1688

Received 17th January 2019, Accepted 7th May 2019

DOI: 10.1039/c9mh00094a

rsc.li/materials-horizons

\section{Negative piezoelectric effect in an organic supramolecular ferroelectric $\uparrow$}

\author{
Indre Urbanaviciute, (D) ${ }^{a}$ Xiao Meng, ${ }^{b}$ Michal Biler, (D) ${ }^{c}$ Yingfen Wei, (D) ${ }^{d}$ \\ Tim D. Cornelissen, (iD a Subham Bhattacharjee, $\ddagger^{b}$ Mathieu Linares iD ef and \\ Martijn Kemerink (D) *a
}

The vast majority of ferroelectric materials demonstrate a positive piezoelectric effect. Theoretically, the negative piezoelectric coefficient $d_{33}$ could be found in certain classes of ferroelectrics, yet in practice, the number of materials showing linear longitudinal contraction with increasing applied field $\left(d_{33}<0\right)$ is limited to few ferroelectric polymers. Here, we measure a pronounced negative piezoelectric effect in the family of organic ferroelectric small-molecular BTAs (trialkylbenzene-1,3,5-tricarboxamides), which can be tuned by mesogenic tail substitution and structural disorder. While the largeand small-signal strain in highly-ordered thin-film BTA capacitor devices are dominated by intrinsic contributions and originates from piezostriction, rising disorder introduces additional extrinsic factors that boost the large-signal $d_{33}$ up to $-20 \mathrm{pm} \mathrm{V} \mathrm{V}^{-1}$ in short-tailed molecules. Interestingly, homologues with longer mesogenic tails show a large-signal electromechanical response that is dominated by the quadratic Maxwell strain with significant mechanical softening upon polarization switching, whereas the small-signal strain remains piezostrictive. Molecular dynamics and DFT calculations both predict a positive $d_{33}$ for defect-free BTA stacks. Hence, the measured negative macroscopic $d_{33}$ is attributed to the presence of structural defects that enable the dimensional effect to dominate the piezoelectric response of BTA thin films.

\footnotetext{
${ }^{a}$ Complex Materials and Devices, Department of Physics, Chemistry and Biology (IFM), Linköping University, 58183 Linköping, Sweden. E-mail: martijn.kemerink@liu.se

${ }^{b}$ Laboratory of Macromolecular and Organic Chemistry, Eindhoven University of Technology, P.O. Box 513, 5600 MB Eindhoven, The Netherlands ${ }^{c}$ Department of Chemistry - BMC,

Uppsala University, Box 576, Uppsala S-751 23, Sweden

${ }^{d}$ Zernike Institute for Advanced Materials, University of Groningen, 9747 AG Groningen, The Netherlands

${ }^{e}$ Department of Science and Technology (ITN), Campus Norrköping, Linköping University, SE-60174 Norrköping, Sweden

${ }^{f}$ Swedish e-Science Research Centre (SeRC), Linköping University,

SE-581 83 Linköping, Sweden

$\dagger$ Electronic supplementary information (ESI) available. See DOI: 10.1039/ c9mh00094a

\$ Present address: Department of Chemistry, Kazi Nazrul University, Asansol, Burdwan, 713340, West Bengal, India.
}

\section{New concepts}

Materials with a negative piezoelectric effect demonstrate atypical, inverse strain-field and polarization-stress characteristics. While the mechanisms driving the conventional - positive - piezoelectricity are transparent and manifest in a plethora of materials, negative piezoelectricity requires special conditions and was only found in two-phased (i.e. coexisting crystalline and amorphous matter) semi-crystalline ferroelectric polymers, with the renowned (and so far only) successfully utilized example of P(VDF-TrFE). Here, we experimentally demonstrate a pronounced negative piezoelectric effect in a whole family of organic small-molecular ferroelectric materials that can be varied with tail-substitution. The well-defined, robust and tunable columnarhexagonal (liquid)crystalline nanostructure of these self-assembling materials allows precise control of the macroscopic polarization and of the level of structural disorder in the layers. Using this tunability we disclose a counterintuitive, significant growth of the large-signal piezoelectric response with increasing structural disorder, while the small-signal response remains unaffected. Mechanical softening upon polarization switching leads to a unique amplification of the large-signal electromechanical response of longer-substituted compounds. The reported high piezoelectric coefficients, long polarization retention, low fatigue, great temperature and environmental stability accompanied by mechanical softness makes BTA a perspective candidate material for application in flexible transducers and energy harvesters.

\section{Introduction}

Since the discovery of the piezoelectric effect in the last decades of the 19th century, a great number of both natural ${ }^{1,2}$ and synthetic $^{3,4}$ materials have been found to demonstrate piezoelectric response. The property of these materials to convert mechanical energy to electrical energy (direct piezoelectric effect) and vice versa (converse piezoelectric effect) has found wide-ranging applications, from energy harvesters to transducers and beyond. ${ }^{5-7}$ The key materials in the field remain the inorganic ferroelectric perovskites like BTO and PZT. Despite environmental concerns (toxicity, high sintering temperatures, etc.), complex device fabrication procedures and limiting physical properties (e.g. high density, brittleness), these ceramic materials have been used extensively due to their exceptionally high 
ferroelectric and piezoelectric coefficients. Current advancement in the field of organic ferroelectrics and piezoelectrics has led to materials having comparable parameters, including remnant polarizations of up to $230 \mathrm{mC} \mathrm{m}^{-2}$ in single crystals of DIPAB and croconic acid, ${ }^{9}$ piezoelectric constants reaching $350 \mathrm{pm} \mathrm{V}^{-1}$ in antiferroelectric TFMNI crystals, ${ }^{10} 40 \mathrm{pm} \mathrm{V}^{-1}$ in imidazolium perchlorate thin films, ${ }^{11,12}$ above $-60 \mathrm{pm} \mathrm{V}^{-1}$ in $\mathrm{P}(\mathrm{VDF}-\mathrm{TrFE})$ devices, ${ }^{13-15}$ and others. ${ }^{16-18}$ In addition, organic ferro- and piezoelectric materials are typically non-toxic, lightweight, flexible and soft, but most importantly, due to good thin-film forming properties, organic material devices can in many cases be fabricated using cheap and efficient processes. Because of this, organic ferroelectrics may become relevant for a wide variety of applications like conformal and/or flexible sensors, actuators and energy harvesters for which inorganic ferroelectrics are unsuited and can be outperformed. ${ }^{19}$ Yet, the new materials are rarely characterized in real-device conditions, and their piezoelectric properties are typically studied in bulk single-crystals or using controversial piezoresponse force microscopy (PFM). ${ }^{20}$ Due to this, with a few notable exceptions, the macroscopic piezoelectric properties of organic ferroelectrics are only sparsely known and far from completely understood.

The total longitudinal strain $S_{33}$ in ferroelectric layers arises from the electrostriction coupled to the electric displacement $D$ and can be expressed as: ${ }^{14}$

$S_{33}=Q_{33} D^{2}=Q_{33}\left(\varepsilon_{\mathrm{r}} \varepsilon_{0} E+P_{\mathrm{r}}\right)^{2}=Q_{33}\left(\varepsilon_{\mathrm{r}} \varepsilon_{0} E\right)^{2}+Q_{33} P_{\mathrm{r}}^{2}+2 Q_{33} \varepsilon_{\mathrm{r}} \varepsilon_{0} P_{\mathrm{r}} E$

The electrostriction due to the applied field has a quadratic field dependence $S_{\mathrm{d}}=Q_{33}\left(\varepsilon_{\mathrm{r}} \varepsilon_{0} E\right)^{2}$ and is characteristic to all dielectrics. In organic ferroelectrics like $\mathrm{P}(\mathrm{VDF}-\mathrm{TrFE})$ the effect is very weak, resulting in $<0.005 \%$ strain. The ferroelectric polarization-induced electrostriction, or piezostriction, $S_{\mathrm{P}}=\left(2 Q_{33} \varepsilon_{\mathrm{r}} \varepsilon_{0} P_{\mathrm{r}}\right) E=d_{33} E$ underlies the common linear piezoelectric effect in otherwise spontaneously strained $S_{\mathrm{r}}=Q_{33} P_{\mathrm{r}}{ }^{2}$ ferroelectric layers. The electrostriction coefficient $Q_{33}$ is inversely proportional to the elastic stiffness constant $Y_{33}$ (Young's modulus) through $Q_{33}=$ $\gamma_{33} / Y_{33}$, where $\gamma_{33}$ is the electrostrictive coupling parameter.

Piezoelectric response in (poly)crystalline materials typically originates from field-induced lattice deformations, as due to the applied electric field the lattice dipoles stretch, resulting in longitudinal expansion, and vice versa. ${ }^{21}$ For small deformations this gives a linear strain-field (or polarization-stress) dependence with positive longitudinal electrostriction $Q_{33}$ and piezoelectric $d_{33}$ coefficients. An inverted response - contraction with increasing field and negative $Q_{33}, d_{33}$ coefficients - is therefore rare, because it requires different, unconventional mechanisms to be at play. For idealized $S-E$ butterfly loops characteristic to each case see Fig. S1 $(\mathrm{ESI} \dagger)$. A recent data-mining study has revealed that the negative piezoelectric coefficient should theoretically be found in a large number of ABC-type inorganic ferroelectrics. ${ }^{22}$ However, experimentally the negative $d_{33}$ has been observed principally in organic semi-crystalline ferroelectric polymers, with the renowned example of the $\mathrm{P}(\mathrm{VDF}-\mathrm{TrFE})$ seen in Fig. 1b, or their composites. ${ }^{23-25}$ Biopolymers like cellulose or keratin have been shown to have negative piezoelectric constants for shear $\left(d_{14}<0\right)$ with longitudinal ones being negligible, ${ }^{26}$ while soft tissues comprising ferroelectric proteins, e.g. aortic walls, have negative electrostriction coefficients yet are likely not piezoelectric. ${ }^{27}$ The key to the negative longitudinal piezoelectricity in these materials is the coexistence of two phases of different elastic compliance, with a condition that the stiffer of the two is ferroelectric with the polarization in the thickness direction while the softer is anisotropically deformable (with Poisson ratio $\nu<0.5$ ). This leads to coupling between the dipole density and the macroscopic dimensions of the sample and has been shown to (a)

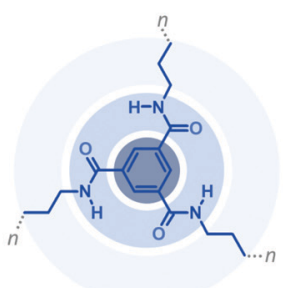

$\pi$-stacking benzene core $\mathrm{H}$-bonding dipolar amide group Flexible alkyl tail

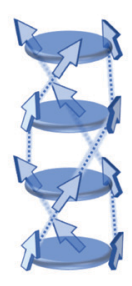

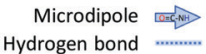

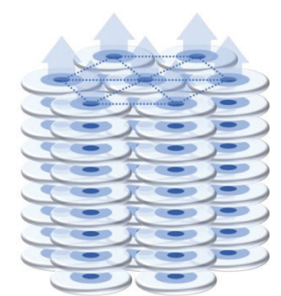

Macrodipole

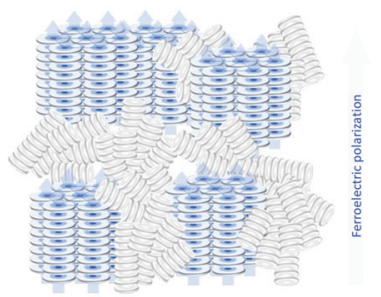

(b)
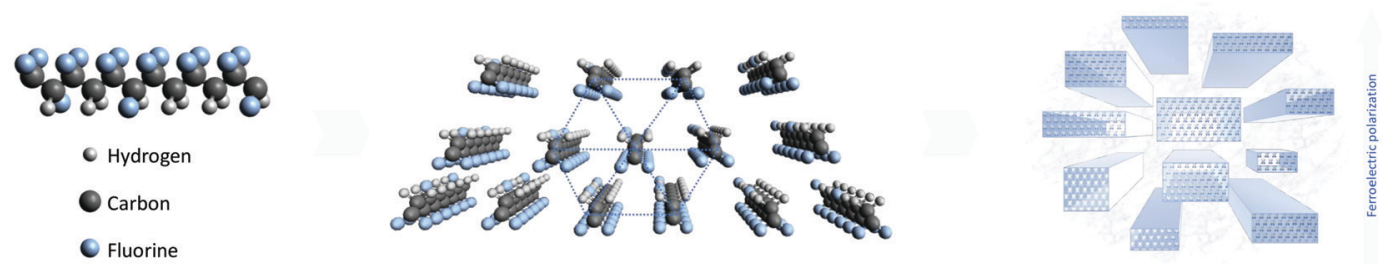

Fig. 1 Structure and morphology of BTAs and P(VDF-TrFE). (a) Primary, secondary, tertiary and quaternary structure of BTAs. Despite self-organization in a hexagonally packed (liquid)-crystalline phase, real ferroelectric layers have a certain level of structural disorder. (b) P(VDF-TrFE) is a semi-crystalline polymer with crystallinity exceeding $50 \%$. Crystalline lamellae, consisting of pseudo-hexagonally packed polymer chains, are distributed in an amorphous matrix. Illustrations are not to scale. 
underly direct as well as converse piezoelectric response (see Dimensional effect in the ESI $\dagger$ ). ${ }^{13}$

Here, we demonstrate experimentally that the negative longitudinal piezoelectric effect can be found in a small-molecular organic ferroelectric - the trialkylbenzene-1,3,5-tricarboxamide (BTA). BTA is a columnar-hexagonal (liquid)-crystal, see Fig. $1 \mathrm{a},{ }^{28-30}$ having a remnant polarization of up to $\sim 60 \mathrm{mC} \mathrm{m}^{-2}$, depending on the tail substituent. ${ }^{31}$ Differently to the conventional liquidcrystalline materials used for electrooptical applications, BTA maintains its macrodipole orientation at zero external field due to $\pi-\pi$ stacking and hydrogen-bonding, which allows it to keep the polarization for up to 10 years at room temperature. ${ }^{31-33}$ The overall performance of thin-film BTA capacitor devices is comparable to devices based on $\mathrm{P}(\mathrm{VDF}-\mathrm{TrFE})$, additionally offering some advantages like lower processing temperatures, easy chemical tuning and a wider operational temperature window. ${ }^{31,34,35}$ In this study we present interferometrically measured converse piezoelectric response in solution-processed thin-film BTA capacitor devices. Using density functional theory (DFT) and molecular dynamics (MD) simulations, we explain the origin of the observed anomalous negative piezoelectric effect in BTA from the microscopic and macroscopic perspective. The observed differences in small-signal and large-signal piezoelectric response further disclose different underlying mechanisms that are strongly influenced by the level of structural disorder in the active layer. The main finding of the study - the negative $d_{33}$ in small-molecular materials - sheds important new insight on a phenomenon that was previously considered a peculiarity of a specific polymer ferroelectric, $\mathrm{P}(\mathrm{VDF}-\mathrm{TrFE})$. A new prospective class of organic ferroelectric materials with a negative longitudinal piezoelectric constant is unearthed, that includes but is not limited to the amide-based discotic mesophases.

\section{Results}

\section{Typical large-signal and small-signal piezoelectric characteristics of BTA-C6}

We measured the converse piezoelectric effect in thin-film spincoated capacitor devices of BTAs using a double-beam laser interferometer system (DBLI), ${ }^{36}$ coupled with a voltage signal generator, high-voltage amplifier and a multifunctional output analyzer. This allowed for concurrent obtention of the strain and polarization characteristics at large-signal, as well as of the piezoelectric coefficient, phase and capacitance at small-signal measurement conditions. The DBLI system allows to reliably probe the piezoelectric response at real-device conditions (with electrodes) with minimal intervention. For further details see the Methods section and ESI. $\dagger$ All measurements were conducted at $60{ }^{\circ} \mathrm{C}$ to allow comparison of different BTA homologues, as will be discussed in detail further in the text. The devices were characterized in 33 mode, i.e. the strain, the polarization and the applied electric field were on the same $z$ axis.

Typical large-signal strain-field butterfly loops measured for short-tailed BTA-C6 devices have a close-to-ideal ( $c f$. Fig. S1, ESI $\dagger$ ) yet anomalous 'flipped' form, see Fig. 2a: the layer expands when the applied field polarity is opposite to the polarization and contracts when electric field and polarization directions match. The reversal occurs around the coercive field. The $S-E$ dependence is linear and has a prominent negative slope, which indicates a negative piezoelectric coefficient, as $S_{33}=d_{33} E$. From a strain-displacement $(S-D)$ and straindisplacement squared $\left(S-D^{2}\right)$ representation of the data (see Fig. S3(a-c), ESI $\dagger$ ), based on fits to eqn (1), negative values for the electrostriction coefficient $Q_{33}$ were obtained, which varied in range -0.55 to $-1 \mathrm{~m}^{4} \mathrm{C}^{-2}$ for BTA-C6, depending on the chosen fitting segment. To compare, -0.8 to $-1.5 \mathrm{~m}^{4} \mathrm{C}^{-2}$ was found in our P(VDF-TrFE) devices, see Fig. S3(d-f) (ESI $\dagger)$. Based on the description of the piezoelectric constant $d_{33}=2 Q_{33} \varepsilon_{\mathrm{r}} \varepsilon_{0} P_{\mathrm{r}}$, $Q_{33}=-1 \mathrm{~m}^{4} \mathrm{C}^{-2}$ obtained for this typical BTA-C6 device leads to $d_{33} \approx-7 \mathrm{pm} \mathrm{V}^{-1}$ with $\varepsilon_{\mathrm{r}}=7$ (from Fig. S4c, ESI $\dagger$ ), which matches the linear slope of $-7 \mathrm{pm} \mathrm{V}^{-1}$ and suggests that polarization-induced electrostriction (eqn (1)) is the dominant factor in the piezoelectric behavior. Closely matching values of all piezoelectric coefficients $d_{33}, e_{33}, g_{33}$ and $h_{33}$ calculated from independent experimental data of $d_{33}, g_{33}$ and $Q_{33}$ (Section II, Table S1 in the ESI $\dagger$ ) further prove the fact. Interestingly, we later show that the large-signal $d_{33}$ can be enhanced to reach
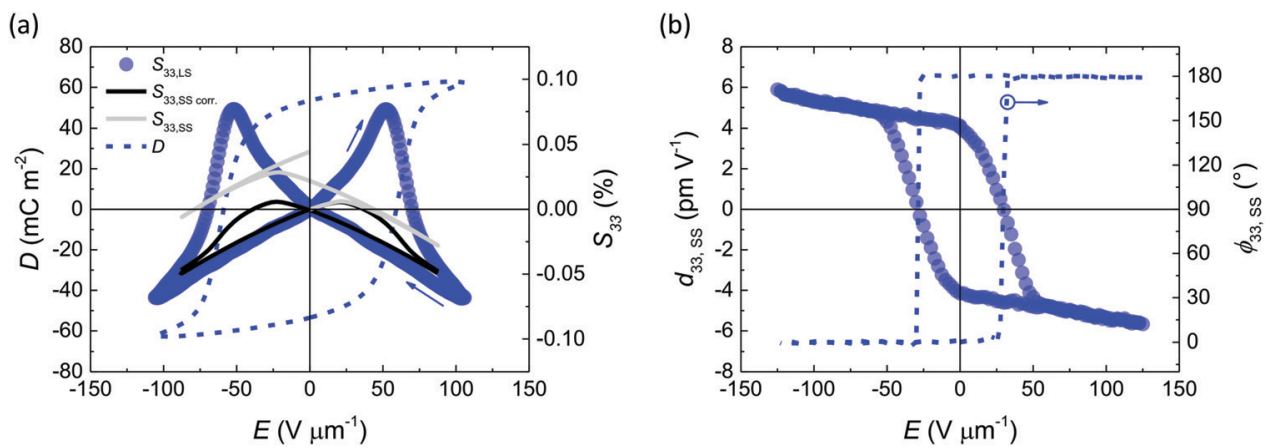

Fig. 2 Typical piezoelectric switching characteristics of BTA-C6. (a) Large- and small-signal strain-field and displacement-field characteristics of BTAC6 thin-film capacitor device as indicated in the legend. BTA-C6 demonstrates a negative longitudinal piezoelectric effect, which is also observed in the piezoelectric coefficient $d_{33}$ and phase $\varphi_{33}$ dependence on the applied field measured at small-signal conditions (b). The small-signal strain (grey line in panel (a)) is obtained by integration of these $d_{33,5 s}-E$ characteristics and corrected for the irreversible polarization effect to get a closed loop (black solid line in panel (a)). 
values as large as $-20 \mathrm{pm} \mathrm{V}^{-1}$, which would correspond to a high $Q_{33} \approx-3 \mathrm{~m}^{4} \mathrm{C}^{-2}$.

The negative piezoelectric effect was observed at small-signal conditions as well, see Fig. 2b. The small-signal piezoelectric coefficient $d_{33, \mathrm{ss}}$, phase $\varphi_{33, \mathrm{ss}}$ and capacitance were measured on the macroscopic device simultaneously using the DBLI, see Methods section and ESI $\dagger$ for details. The measured textbook-like $d_{33, \mathrm{ss}}-E$ hysteresis loop indicates a negative $d_{33}$ at positive applied voltages, which is opposite to the trends observed in conventional inorganic ferroelectrics with the positive piezoelectric effect. ${ }^{37}$ Interestingly, the small-signal $d_{33}$ is lower than the one measured at large-signal conditions: around $-4.5 \mathrm{pm} \mathrm{V}^{-1}$ at zero field for BTA-C6 vs. $d_{33, \mathrm{LS}}=-7 \mathrm{pm} \mathrm{V}^{-1}$ for the same device. A similar mismatch is observed in our $\mathrm{P}(\mathrm{VDF}-\mathrm{TrFE})$ devices with $d_{33, \mathrm{SS}}=-25 \mathrm{pm} \mathrm{V}^{-1}$ compared to $d_{33, \mathrm{LS}}=-38 \mathrm{pm} \mathrm{V}^{-1}$, see Fig. S4(d-f) (ESI $\dagger)$.

To find an explanation for this discrepancy one must consider the differences in switching mechanisms at small- and large-signal conditions. While the large-signal response is considered to be governed by both reversible and irreversible polarization switching processes, the small-signal effects are mostly driven by reversible polarization modulations. ${ }^{37}$ Irreversible changes are related to extrinsic phenomena as transitions between global energy minima $\left(\right.$ e.g. $+P_{\mathrm{r}}$ and $\left.-P_{\mathrm{r}}\right)$ and movement of ferroelectric domain walls; reversible effects rise from the dielectric response and intrinsic phenomena that are coupled to small fluctuations around local minimum potential points, such as ion displacement in perovskite ferroelectrics, or, in the BTA case, columnar macrodipole rotation, as illustrated in the inset of Fig. 3c.

Considering a single self-assembled molecular stack, one would expect a positive piezoelectric response at the nanoscale due to the applied field rotating the amide dipoles further out-of-plane, which does not agree with the measured macroscopic negative piezoelectric constants. To this end, we have simulated the behavior of a single molecular stack consisting of 18 methyl-tailed BTAs using the energy minimization principle in molecular dynamics ${ }^{33}$ and found that with increasing field, (a)

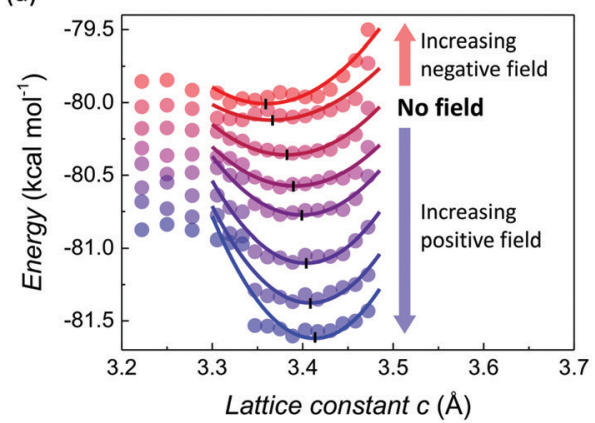

(c)

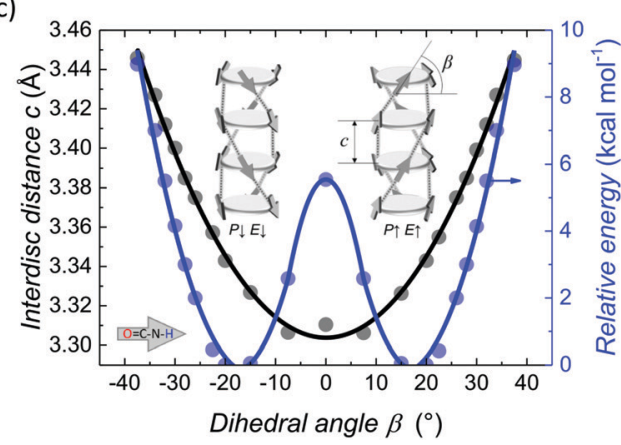

(b)

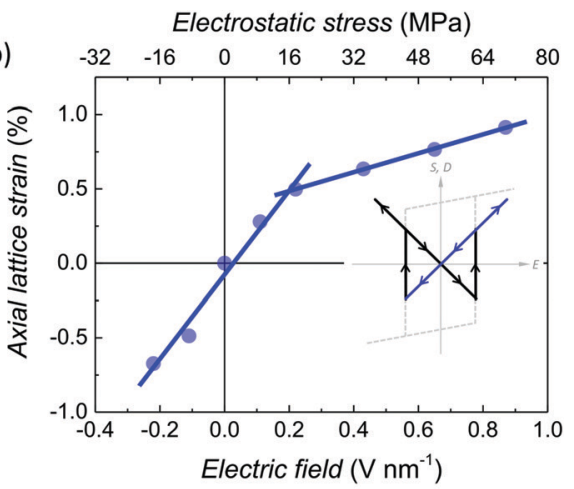

(d)

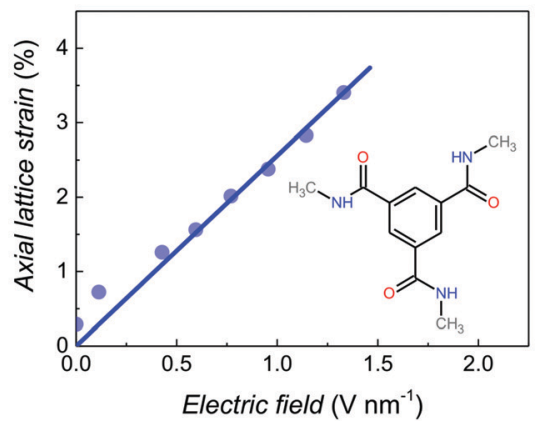

Fig. 3 Simulated field-induced axial lattice strain. (a) Energy per molecule, including both van der Waals and electrostatic interactions, for a single molecular BTA-C1 stack versus lattice constant parametric in external electric field, obtained from molecular dynamics (MD) simulations. The optimal (minimum energy) axial lattice constant is marked by a vertical bar. The lines are parabolic fits to the MD data (dots). (b) Extracted axial lattice strain

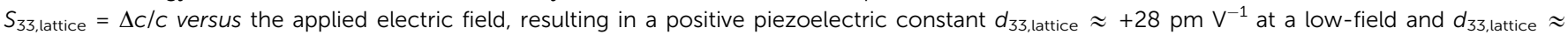
$+6 \mathrm{pm} \mathrm{V}^{-1}$ at a high-field regime. The simulation results correspond to the upper positive segment (i.e. after positive poling) of the strain-field butterfly loop, as illustrated in the inset. Electric field-induced stress (top horizontal axis) versus lattice strain trend indicates mechanical stiffening of the lattice in

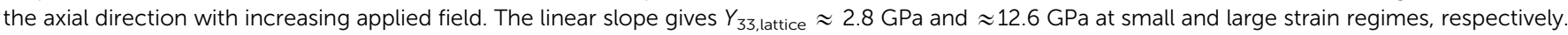
(c) Energy profile of macrodipole switching determined at B3LYP-D3/cc-pVDZ level of theory for a BTA-C1 dimer. The intermolecular distance is calculated between the phenyl moieties of the dimer. Solid lines are guides to the eye. (d) Longitudinal lattice strain versus field characteristic calculated from the data in panel (c). The slope of the trend gives a positive nanoscopic piezoelectric constant $d_{33} \approx+25$ pm $V^{-1}$. Molecular structure of the studied system is given in the inset of panel (d). UP ( $P \uparrow$, right) and DOWN ( $P \downarrow$, left) conformations of self-assembled BTA columnar macrodipoles formed by the helical hydrogen-bonded system of microdipoles with a characteristic dihedral angle of the amide moieties $\pm \beta$ and an interdisc (intermolecular) distance $c$ are illustrated in the inset of panel (c). Side chains are omitted for clarity. 
the longitudinal (interdisc) lattice constant $c$ increases, see Fig. 3a. The columnar macrodipole (inset of panel (c)) stretches when placed in an electric field, corresponding to a positive piezoelectric coefficient at the nanoscale. A linear fit of the axial lattice strain versus field trend gives a positive $d_{33 \text {, lattice } \approx}$ $+28 \mathrm{pm} \mathrm{V}^{-1}$, see panel (b). Mechanical hardening of the lattice is observed at higher fields, which leads to a decreased $d_{33 \text {,lattice }} \approx+6 \mathrm{pm} \mathrm{V}^{-1}$. The inclination of the calculated stress-strain trend in panel (b), top and left axis, respectively, gives a simulated Young's modulus for a single BTA-C1 mole-

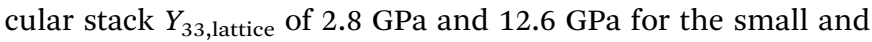
large strain regimes, respectively. The electrostatic stress $X$ here is calculated as the electric force $F$ over the stack area $A: X=$ $F / A=E \cdot Q / A=E \cdot P$, where $E$ is electric field, $Q$ is total charge and $P$ is ferroelectric polarization. The small-strain elastic modulus matches experimentally measured values on single-crystal nanorods of different short-tailed BTAs. ${ }^{38}$ For the longer-tailed BTA-C6, due to lower dipole density, the calculated Young's moduli decrease to 1.9 GPa (small-strain) and 8.6 GPa (large-strain). Based on Hooke's law, the notably decreasing width of the parabolic energy landscape in the panel (a) with increasing electric field indicates a growing Young's modulus (through a larger spring constant), as well. The observation of the non-constant Young's modulus will be relevant in our further discussion, see Electromechanical response of other BTA homologues.

We confirmed the positive nanoscopic $d_{33}$ at a higher level of theory - by quantum-mechanical DFT computations on a selfassembled BTA dimer, see Fig. 3(c and d). BTA columnar macrodipoles (i.e. a helical hydrogen-bonded system of microdipoles) have two preferred orientations UP ( $P \uparrow$, right) or DOWN $(P \downarrow$, left) with a characteristic dihedral angle $\pm \beta$ between the amide moieties and the benzene plane, and an interdisc distance $c$, see inset of panel (c). By DFT simulations, the dihedral angle versus interdisc distance relation and the corresponding characteristic potential energy $U$ can be computed. We performed a scan at the QM level to determine this relation for a BTA-C1 (methyl sidechains, see inset of panel (d)) dimer during its macrodipole $M$ flip from "up" to "down" through a planar conformation of the amide moieties (i.e. dihedral angle $\beta=0^{\circ}$ ) with respect to the phenyl group, see panel (c). This data can be transformed to the longitudinal lattice strain $S_{33 \text {,lattice }}=\Delta c / c$ and the electric field $E=U / M$, as given in panel (d). A change in magnitude of the macrodipole $M$ with the dihedral angle is taken into account. ${ }^{18}$ If we fit this trend, its linear slope (solid line) directly gives the nanoscopic piezoelectric constant $d_{33 \text {, lattice }} \approx$ $+25 \mathrm{pm} \mathrm{V}^{-1}$, which matches the results obtained by molecular dynamics simulations above. Returning to the Young's modulus estimation, DFT calculations using electrostatic stress approach (see Fig. S5a, ESI $\dagger$ ) give lower estimates for both BTA homologues at high-field range, being $3.1 \mathrm{GPa}$ and $4.1 \mathrm{GPa}$ for BTA-C6 and BTA-C1, respectively. For BTA-C12 with dodecyl sidechains this coefficient equals $1.7 \mathrm{GPa}$ which will be important for further discussions. Fig. S5b (ESI $\dagger$ ) compares the elastic moduli of all three BTA homologues obtained by different approaches. More details on both the MD and DFT simulations can be found in the ESI, $\dagger$ "Simulated BTA strain-field trends at the nanoscale".
Despite being very close to the experimentally obtained value in magnitude, the piezoelectric constant $d_{33}$ is of an opposite - positive - sign. In macroscopic devices strictly negative piezoelectric coefficients are observed, which implies that this nanoscopic mechanism is overpowered by other micro- and/or macroscopic phenomena, in particular the dimensional effect as argued throughout the text. Coexistence of nanoscopic positive and macroscopic negative piezoelectric response is very different from the $\mathrm{P}(\mathrm{VDF}-\mathrm{TrFE})$ case, in which negative strain has been reported to arise at all scales - single polymer chains, crystallites and bulk layers (see ESI, $\uparrow$ Dimensional effect for details). However, since the measured real-device macroscopic (large-signal) and microscopic (small-signal) responses in BTA remain negative, we conclude that bulk collective effects, governed by the dimensional effect, preponderate over the nanoscopic single-stack mechanism. In the following we will therefore continue to focus on the different roles of the macroscopic smallsignal and large-signal mechanisms.

To evaluate the relative contributions of reversible-microscopic and irreversible-macroscopic processes, the measured large-signal strain was compared to the small-signal one. The latter was calculated by integration of the small-signal $d_{33}-E$ loops of Fig. 2b. The $S_{33, \mathrm{ss}}-E$ characteristics obtained in this way (grey lines in Fig. 2a) do not give a closed loop. To fully reproduce the measured curve form, extrinsic contributions to the small-signal strain must be taken into account. Based on ref. 37, the smallsignal strain equals $S_{33, \mathrm{SS}}=\int d_{33, \mathrm{SS}} \mathrm{d} E+\Delta S / 2 \cdot \mathrm{ce}_{\mathrm{sw}}$, where $\Delta S$ is the mismatch offset of the $\int d_{33, \mathrm{SS}} \mathrm{d} E$ integral and $\mathrm{ce}_{\mathrm{sw}}$ is the fraction of the switched cells. $\mathrm{ce}_{\mathrm{sw}}$ can be approximated by the irreversible polarization loop, normalized from 0 to 1 . The irreversible polarization (black solid lines in Fig. S4b, ESI $\dagger$ ) can be obtained by subtracting the reversible contribution to the polarization (black dashed line) from the total polarization hysteresis curve (red solid line). For this, the reversible polarization is acquired by integration of the small-signal capacitance $C-V$ butterfly loop (Fig. S4c, ESI $\dagger$ ). It is dominated by a linear dielectric response and gives only slight hysteresis with polarization values lower than $0.1 \mathrm{mC} \mathrm{m}^{-2}$ for BTA-C6. Similar procedure was performed on $\mathrm{P}(\mathrm{VDF}-\mathrm{TrFE})$ and the results are given in Fig. $\mathrm{S} 4(\mathrm{~d}-\mathrm{f})(\mathrm{ESI} \dagger)$.

For BTA-C6 this offset-corrected small-signal strain loop (black solid line in Fig. 2a) reproduces the measured negative large-signal strain quite well, whereas larger deviations are found in the positive strain range. Comparing results for BTA-C6 and P(VDFTrFE), the large-signal strain appears to be dominated by intrinsic (reversible) effects in the former, while extrinsic (irreversible) effects manifest stronger in the latter. This agrees to the results presented in ref. 14 on $\mathrm{P}(\mathrm{VDF}-\mathrm{TrFE})$ claiming that its piezoelectric strain, despite being induced by the reversible lattice deformations, as shown by dynamic in situ XRD measurements, is also influenced by macroscopic crystalline-amorphous matter interactions. Below, we will show that in BTA-C6 the proportion of intrinsic and extrinsic contributions can be controllably tuned.

\section{$d_{33} v s$. remnant polarization and disorder}

BTA molecules self-assemble to form long supramolecular stacks, see AFM micrographs in Fig. S2 (ESI $\dagger$ ). In spin-coated 
thin-films these molecular columns initially lie in-plane and, to be able to operate in the out-of-plane device structure, molecules are aligned homeotropically by a short field-annealing procedure as discussed in the ESI. $\dagger^{30,31}$ The possibility to control the molecular alignment allows to tune the macroscopic remnant polarization and the amount of the nonswitchable material. Thus, to further study the differences in small- and large-signal piezoelectric response we changed the molecular alignment level of our BTA-C6 devices. First, a $d_{33}$ dependence on the macroscopic remnant polarization could be established in this way. Second, if the macroscopic piezostriction-dimensional effect (for crystalline matter in an amorphous matrix) is the dominant factor in the electromechanical response of BTA, a larger fraction of (supposedly) softer nonaligned material should lead to an increase in the large-signal strain and $d_{33}$ through a decrease of the elastic modulus of the bulk $Y_{33}$, as $d_{33}=2 \varepsilon_{\mathrm{r}} \varepsilon_{0} P_{\mathrm{r}} \gamma_{33} / Y_{33}$. The small-signal response would experience a linear dependence on the fraction of aligned material, i.e. $P_{\mathrm{r}}$, as, based on the discussion above, it is mostly driven by intrinsic microscopic effects.

During the homeotropic alignment procedure, the temporal growth of the remnant polarization is of cumulative Gaussian fashion with clear saturation (see Fig. 4) and most likely proceeds via a nucleation-and-growth mechanism. Different levels of remnant polarization can be obtained by stopping the alignment at different stages and cooling to the measurement temperature. The results of the measured dependence show that both the piezoelectric coefficient and the strain decrease in magnitude with lower molecular alignment level, see Fig. S6 (ESI $\dagger$ ) for details. A linearly growing $d_{33}$-polarization dependence is found for both the large- and small-signal response with a slope of around $-0.15 \mathrm{pm} \mathrm{V}^{-1}$ and $-0.085 \mathrm{pm} \mathrm{V}^{-1}$ per $1 \mathrm{mC} \mathrm{m}^{-2}$ respectively. The disorder parameter (vide infra) was nearly independent of alignment level. To compare, a slope of $-0.55 \mathrm{pm} \mathrm{V}^{-1}$ per $1 \mathrm{mC} \mathrm{m}^{-2}$ at large-signal conditions was previously found for $\mathrm{P}(\mathrm{VDF}-\mathrm{TrFE})$ with $P_{\mathrm{r}}$ controlled by the annealing temperature. ${ }^{14,39}$ These results clearly show that the piezoelectric response cannot be improved by changing the alignment level. This, and the approximately linear dependence on $P_{\mathrm{r}}$, point to $d_{33}$ being dominated by microscopic effects in well-aligned BTA-C6.
We discovered that another way to tune the piezoelectric coefficients is through the layer disorder. Structural disorder of a ferroelectric arises from various defects and manifests itself as a distribution in local coercive fields, as stated by the classical Preisach theory. ${ }^{40}$ In a recent work we presented an experimental approach to measure this Preisach distribution (PD) and established a direct connection between the material's morphology and the width and shape of the PD. ${ }^{41}$ However, to evaluate the width of the distribution, a projection of the PD is sufficient, which is straightforwardly obtained as the switching current response to a triangular voltage signal, i.e. the derivative of the macroscopic polarization hysteresis loop. ${ }^{42}$ Having all the hysteresis loops measured, we fitted the corresponding $J-V$ curves to a Gaussian function, see Fig. S8a (ESI $\dagger$ ) for examples. To be able to compare distributions of different devices with different parameters we normalized these to the coercive field value on the abscissa axis and the maximum hysteron (i.e. switching current) density on the ordinate axis. The extracted standard deviation $\sigma$ was used as a proxy for structural disorder and the amount of amorphous material present in the active layer. ${ }^{41}$ The obtained $\sigma$ parameter varied in range $0.1-0.45$ for BTA-C6. The measurements were performed at $60{ }^{\circ} \mathrm{C}$ to compare the behaviour among the BTA homologues (see below), at which they all are in the columnar-hexagonal liquid-crystalline state and ferroelectrically switchable (see ESI $\dagger$ ). ${ }^{31}$

A surprisingly clear disorder dependency is found among BTA-C6 devices. As seen in Fig. 5a, the small-signal $d_{33}$ (black open symbols) slightly deteriorates from around $-4.7 \mathrm{pm} \mathrm{V}^{-1}$ to $-3.5 \mathrm{pm} \mathrm{V}^{-1}$ with disorder. In contrast, the large-signal $d_{33}$ has a strong growing trend from $-5 \mathrm{pm} \mathrm{V}^{-1}$ to $-21 \mathrm{pm} \mathrm{V}^{-1}$. This strong $d_{33 \text {,Ls }}$ correlation with the disorder parameter causes the datapoint scattering in the remnant polarization dependence of panel (b) of Fig. 5 as devices with equal $P_{\mathrm{r}}$ can have different disorders. For polarization-dependent data with constant disorder see Fig. S6 (ESI $\dagger$ ). Comparing panels (a) and (b), the large-signal response is noticeably disorder-dominated, while the small-signal response is more sensitive to the remnant polarization value. This accords well with the previously discussed macroscopic-extrinsic-irreversible and microscopicintrinsic-reversible switching processes and suggests that (a)

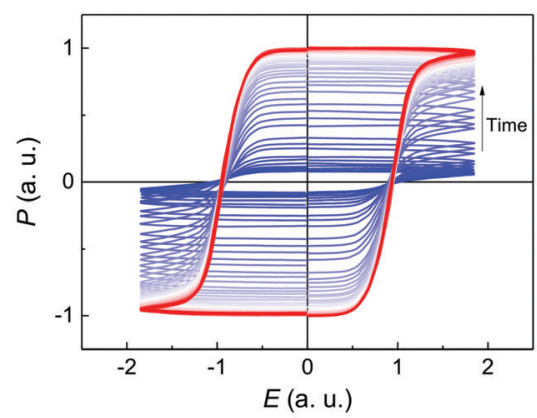

(b)

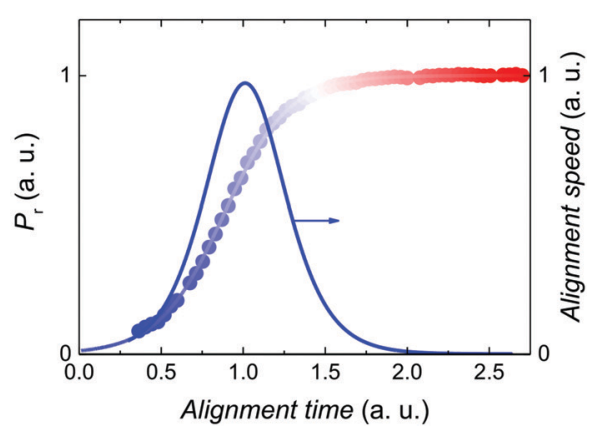

Fig. 4 Typical homeotropic alignment procedure of BTAs. (a) Gradual growth of remnant polarization is measured by bipolar voltage cycling during the field-annealing alignment process. (b) The temporal polarization growth is of Gaussian fashion with a clear saturation. Precise control of the macroscopic remnant polarization can be achieved by stopping this alignment procedure at a desired level. 
(a)

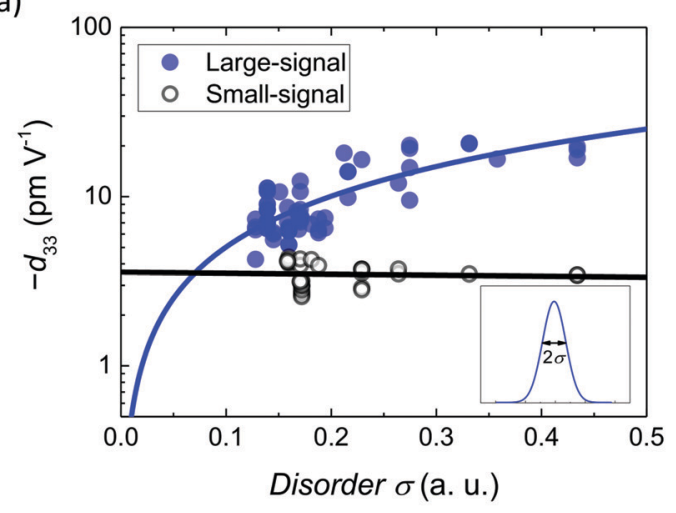

(b)

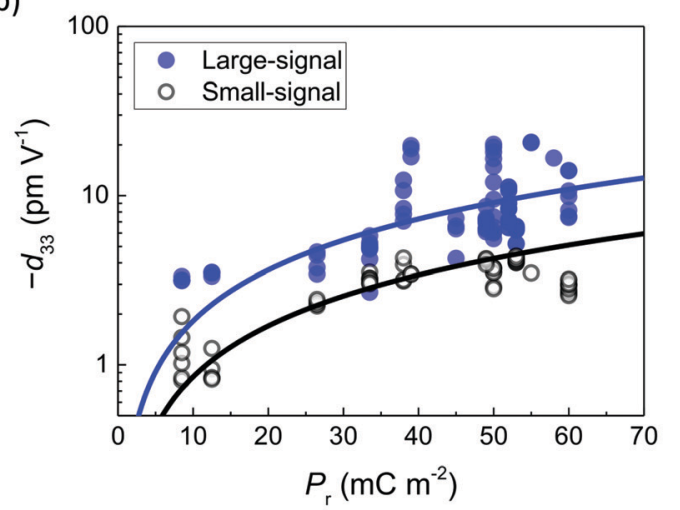

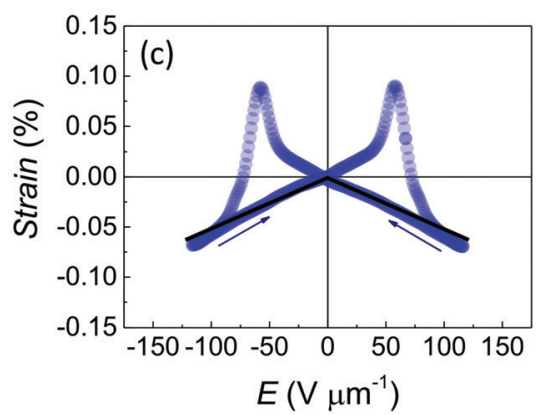
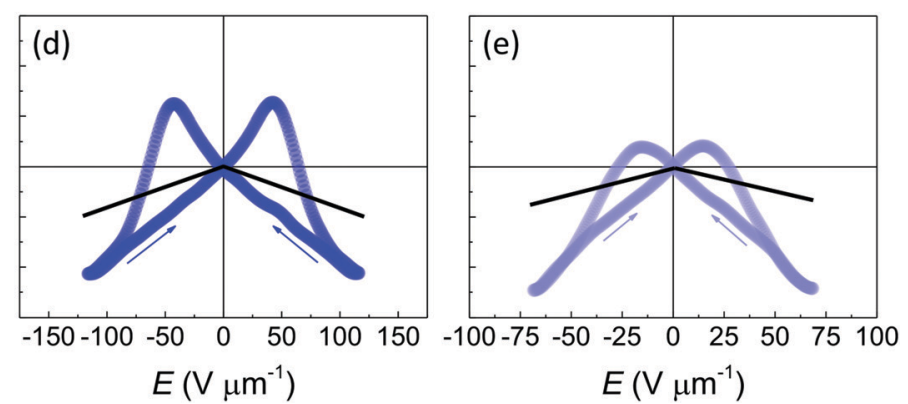

Fig. 5 Tuning the piezoelectric coefficients of BTA-C6. Large-signal (blue full symbols) and small-signal (black open symbols) piezoelectric coefficient $d_{33}$ dependence on (a) the relative energetic disorder $\sigma$ (for varying $P_{\mathrm{r}}$ ) which was obtained from fitting of the normalized switching current transients to a Gaussian function (see inset), and on (b) the remnant polarization (for varying disorder). Polarization and disorder of BTA-C 6 devices were controlled as explained in the text. Solid lines are linear fits. Semi-logarithmic scale is used to better represent the small $d_{33}$ values. (c-e) Examples of large-signal strain-field butterfly loops (blue symbols) for low, medium and large disorder, respectively. Black lines give a slope of corresponding small-signal characteristics (see Fig. S8, ESI ).

disorder is a macroscopic (bulk) characteristic. The disorder influences the form of the strain-field butterfly loops, similarly to its slanting effect on the polarization hysteresis loops. Fig. 5(c-e) shows that for the least disordered devices closeto-ideal $S-E$ hysteresis loops with small mismatch between the large- and small-signal strain are found, whereas blunt elongated loops, with large mismatch between the largeand small-signal strain, are found for the most disordered ones. Thus, with the help of disorder, the balance between the intrinsic and extrinsic polarization switching contributions is gradually moved towards the extrinsic (irreversible effects) side.

To summarize our findings so far, we have shown that BTA-C6 demonstrates a negative longitudinal piezoelectric effect, which can be tuned by the macroscopic remnant polarization and the level of disorder. The piezostriction (polarizationcoupled electrostriction) rationalizes the observed linear strainfield dependence, and the dimensional effect determines the negative sign of its slope, $d_{33}$. For highly-ordered devices both the small- and large-signal piezoelectric responses arise from the intrinsic reversible contributions. For more disordered layers extrinsic irreversible effects begin to dominate the large-signal strain, which boosts the $d_{33}$ values to $-20 \mathrm{pm} \mathrm{V}^{-1}$ and above. This result is roughly twice lower than that of the $\mathrm{P}(\mathrm{VDF}-\mathrm{TrFE})$, yet it surpasses a large number of organic ferroelectric crystals. ${ }^{18}$ Despite falling behind the best inorganic piezoelectrics in terms of $d_{33}$ by an order of magnitude, the material is competitive in terms of the figure of merit for energy harvesting $d g$ due to its relatively high $g_{33}=-0.1 \mathrm{~m}^{2} \mathrm{C}^{-1}$. Based on this combination of the piezoelectric charge and voltage coefficients $d_{33} g_{33}=2 \times 10^{-12} \mathrm{~m}^{3} \mathrm{~J}^{-1}$, BTA-C6 outperforms a number of inorganic and composite piezoelectric materials. ${ }^{6,19}$ The selfhealing quality inherent to liquid-crystals and low electrical fatigue (see Fig. S9, ESI $\dagger$ ) are additional advantages. Another important constant - the electromechanical coupling coefficient $k_{33}=\frac{d_{33}}{\sqrt{\varepsilon_{33} S_{33}}} \approx 0.1$ is similar to quartz. ${ }^{6}$

\section{Electromechanical response of other BTA homologues}

The BTA-C6 molecular core has three short hexyl tails attached to it that determine the relatively short intercolumnar distance $\sim 1.55 \mathrm{~nm}$. BTA-C6 is in a plastic-crystalline (Fig. S10, ESI $\dagger$ ) phase above and crystalline at room temperature and has a geometrically expected dipole density of around $60 \mathrm{mC} \mathrm{m}^{-2} .^{31}$ As piezoelectric coefficients are coupled to the mechanical compliance, easier deformable materials with the same polarization would in principle be preferred. Therefore, we tested different BTA homologues that have longer peripheral chains: C8, C10, C12 and C18, corresponding to a $\mathrm{C}_{n} \mathrm{H}_{2 n+1}$ alkyl tail. With the molecular core remaining the same, longer flexible alkyl chains bring more intrinsic mobility and thus shift the 
properties to more liquid-crystalline (see a phase diagram in Fig. S10, ESI $\dagger$ ). This also results in higher structural disorder, as previously confirmed by X-ray scattering techniques. ${ }^{43}$ However, due to the longer side-chains, the dipole density gradually decreases from $\sim 45 \mathrm{mC} \mathrm{m}^{-2}$ for BTA-C8 to $\sim 20 \mathrm{mC} \mathrm{m}^{-2}$ for BTA-C18. ${ }^{31}$

Large-signal electromechanical responses for all BTAs are given in Fig. S11 (top row) (ESI $\dagger$ ). Interestingly, while BTA-C8 still demonstrates C6-like negative piezoelectric switching with $d_{33} \approx-8 \mathrm{pm} \mathrm{V}^{-1}$, other homologues have unusual, virtually contraction-only strain-field loops of opposite sense with prominent negative peaks around the coercive field, as shown in Fig. 6a. BTA-C12 is used as an example here and in further analysis. This effect, like the regular negative piezoelectric effect seen in BTA-C6, is not transient and intermediate unsaturated loops fit nicely within the full-saturation curve, see Fig. S12 (ESI $\dagger$ ). If operated on the steepest linear branch segment, the device would give an effective strain-field relation coefficient of more than $-50 \mathrm{pm} \mathrm{V}^{-1}$. While the electrostriction equation $S_{33}=$ $Q_{33} D^{2}$ could reproduce the negative peaks (Fig. S13a, ESI $\dagger$ ) when an (atypical) positive $Q_{33}=+0.55 \mathrm{~m}^{4} \mathrm{C}^{-2}$ was used, the fit could not explain the lack of positive strain. In combination with the Maxwell strain (see below), a higher quality fit is obtained (Fig. S13c, ESI $\dagger$ ). However, such exceptional behavior does not agree with the negative electrostrictive and piezoelectric coefficients discussed above for BTA-C6. Hence, we propose that the Maxwell strain must be considered as the dominant factor in the electromechanical response of the long-substituted BTAs.

Maxwell stress is a well-known effect, present in parallel-plate capacitors due to attractive electrostatic forces acting on the oppositely charged surfaces. This causes negative strain on dielectric spacers, which is directly proportional to the mechanical compliance $(s=1 / Y)$ of the material. For small deformations (where $\frac{F}{A}=Y S$ holds) the stress-strain relation is approximately $S=-\frac{\varepsilon_{\mathrm{r}} \varepsilon_{0}}{2 Y} E^{2}$, with Maxwell stress $\frac{F}{A}=\frac{1}{2} \varepsilon_{\mathrm{r}} \varepsilon_{0} E^{2}$, where $F$ is the compressive force, $Y$ is the Young's modulus, $E$ is the applied field, $\varepsilon_{\mathrm{r}} \varepsilon_{0}$ is the dielectric permittivity of the material and $A$ is the contact area. This effect has been extensively studied and successfully applied in the field of elastomer actuators. ${ }^{44,45}$ The quadratic-to-field Maxwell stress induced strain gave a good fit of the reverse parts of the $S-E$ loops, i.e. after polarization saturation, for a Young's modulus of $Y_{33}=0.75 \mathrm{GPa}$ for BTA-C12 (Fig. S13b, ESI †). A similar procedure resulted in $0.95 \mathrm{GPa}$ for BTA-C10 and $0.3 \mathrm{GPa}$ for BTA-C18 at $60{ }^{\circ} \mathrm{C}$. This trend leads to extrapolated $Y_{33}$ values of $1.2 \mathrm{GPa}$ for BTA-C8 and $1.5 \mathrm{GPa}$ for BTA-C6. It was previously found that for single fibers of crystalline compact-tailed BTAs the elastic constant can reach 2-3 GPa, depending on the tail substituent (lower $Y$ for longer tails),$^{38}$ which makes the obtained values in range 0.3-1.5 GPa for our longer-substituted liquid-crystals very reasonable. No direct $Y$ measurements on liquid-crystalline BTAs are known to us. To compare, the elastic constant of $\mathrm{P}(\mathrm{VDF}-\mathrm{TrFE})$, depending on annealing temperature, varies between $3-4 \mathrm{GPa} .{ }^{46}$ We suggest that this mechanical softness together with the lower remnant polarization of the longer-substituted BTAs is the reason that the strain-field characteristics are dominated by Maxwell strain rather than piezostriction, which is very different from the BTA-C6 and C8 materials. See Fig. S14 and Supplementary methods (ESI $\dagger$ ), Piezostriction vs. Maxwell strain, for a further discussion.

To fit the negative peaks at rising fields, the Maxwell strain equation must be upgraded by including the variation in elastic modulus with field. Alternatively, a similar modulation in $\varepsilon_{\mathrm{r}}$ would have the same effect but would be at odds with the measured near-constant value of $\varepsilon_{\mathrm{r}}$ in e.g. Fig. S4c (ESI $\dagger$ ). It is known that $\mathrm{P}(\mathrm{VDF}-\mathrm{TrFE})$ demonstrates mechanical hardening upon poling - the elastic modulus grows with remnant polarization, ${ }^{39}$ yet the effect is static. A dynamic Young's modulus variation was observed and measured in inorganic polycrystalline films upon polarization switching and was related to $90^{\circ}$-domain switching. ${ }^{47}$ A similar dynamic effect is likely to occur in the BTAs upon polarization switching. With a Gaussian distribution of elastic modulus taken into account (Fig. 6b), a very good fit of these odd-shaped $S-E$ loops is obtained, see Fig. 6a (black line). Here, the minimum in elastic
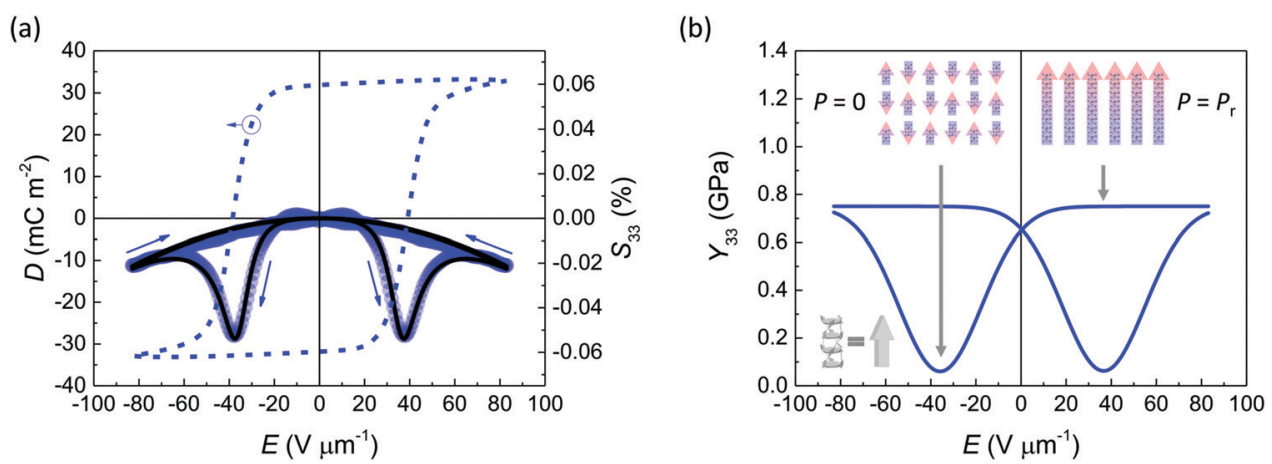

Fig. 6 Large-signal electromechanical response of longer-substituted BTAs. (a) Large-signal displacement-field and strain-field characteristics of BTAC12 (blue dashed line and blue symbols, respectively), also typical for other longer-substituted BTAs. The unusual S-E loop form can be explained by the Maxwell stress induced strain and softening of the material due to polarization switching (black solid line). (b) Young's modulus variation upon polarization switching used for the fitting in panel (a). The maximum $0.75 \mathrm{GPa}$ value is reached when the ferroelectric is fully polarized $P=P_{\mathrm{r}}$, while the minimum $0.06 \mathrm{GPa}$ is found at the randomized polarization point $P=0$, i.e. at the coercive field. Data was obtained at $60{ }^{\circ} \mathrm{C}$. 


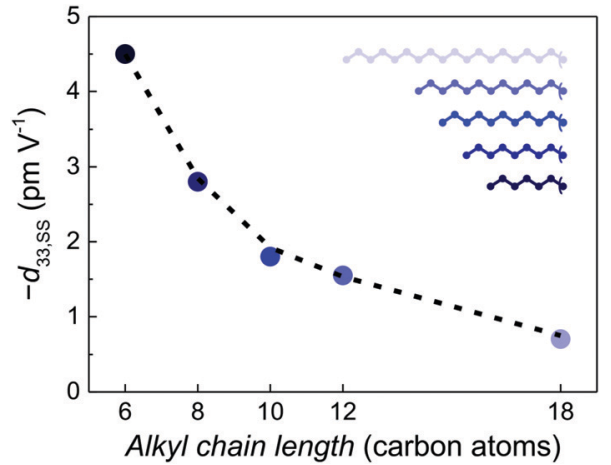

Fig. 7 Maximum experimentally observed small-signal piezoelectric coefficient $d_{33}$ for different BTA homologues. The dashed line represents a trend based on $d_{33}=2 Q_{33} \varepsilon_{\mathrm{r}} \varepsilon_{0} P_{\mathrm{r}}$ with the same constant $Q_{33}=-0.66 \mathrm{~m}^{4} \mathrm{C}^{-2}$ for all homologues.

modulus at $0.06 \mathrm{GPa}$ would be a consequence of the high density of small domains and domain walls at zero net polarization point that leads to a significant weakening of the hydrogen-bonded supramolecular backbone; in contrast, the fully polarized state consists of large, well-developed axial domains, which leads to mechanical hardening and a steady maximum at $0.75 \mathrm{GPa}$. This is illustrated in the inset of Fig. 6b. The small positive strain overshoot around the coercive field of highly-ordered BTA-C6 devices (e.g. Fig. 5c and Fig. S6a, ESI $\dagger$ ) is likely related to the same material softening in combination with the linear piezostriction. The field-induced mechanical stiffening of the lattice in the axial direction found by DFT/MD simulations that was explained earlier in the text agrees well with the discussed experimental finding. The ratio of the perfect-case simulated elastic modulus of $1.7 \mathrm{GPa}$ for BTAC12 (Fig. S5, ESI $\dagger$ ) and the experimental minimum of $0.06 \mathrm{GPa}$ can be used to roughly estimate the fraction of soft amorphous material in the layer, which approaches $\sim 5 \%$.

The small-signal response of longer-substituted BTA homologues remains regular with a negative $d_{33}$, see Fig. S11 bottom row (ESI $\dagger$ ). It is typical for liquid-crystalline materials to maintain short-range order while long-range order is absent. This might well explain why close-to-ideal small-signal $d_{33}-E$ loops are possible despite irregular Maxwell stress dominated largesignal strain. Yet, the small-signal $d_{33}$ decreases significantly with lengthening of the alkyl chains: $-4.5,-2.8,-1.8,-1.55$, $-0.7 \mathrm{pm} \mathrm{V}^{-1}$ for C6, C8, C10, C12 and C18, respectively (Fig. 7). This behavior can be quantitatively reproduced based on the piezostriction formula $d_{33}=2 Q_{33} \varepsilon_{\mathrm{r}} \varepsilon_{0} P_{\mathrm{r}}$ with the same $Q_{33} \approx$ $-0.66 \mathrm{~m}^{4} \mathrm{C}^{-2}$ for all homologues, as shown by the dashed line. Here, we have used typical (measured) remnant polarization $P_{\mathrm{r}}$ and dielectric constant $\varepsilon_{\mathrm{r}}$ values for each homologue.

\section{Discussion}

By combination of interferometric small- and large-signal piezoelectric response measurements we demonstrated a negative longitudinal piezoelectric effect in thin films of the smallmolecular organic ferroelectric BTA (trialkylbenzene-1,3,5tricarboxamide). The piezoelectric coefficient $d_{33}$ was shown to depend strongly on the level of structural disorder in the active layer. While the small-signal $d_{33}$ declined with increasing disorder (from -4.5 to $-3.5 \mathrm{pm} \mathrm{V}^{-1}$ ), an opposite trend was observed under large-signal conditions - significant growth from around $-5 \mathrm{pm} \mathrm{V}^{-1}$ to above $-20 \mathrm{pm} \mathrm{V}^{-1}$. A growing mismatch between small- and large-signal strain with increasing disorder indicates that these responses become governed by different mechanisms. For highly ordered devices, both the small- and large-signal strain are dominated by intrinsic microscopic (reversible) effects and can be explained by pure piezostriction (polarization-coupled electrostriction). With growing disorder, the excessive large-signal strain becomes dominated by extrinsic irreversible factors - domain wall motion and consequent macroscopic polarization switching.

As piezoelectric coefficients are directly proportional to the mechanical compliance, we tested different BTA homologues with longer mesogenic tails and thus stronger expressed liquidcrystalline properties, i.e. higher disorder and lower Young's modulus. Differently to the textbook-like large-signal strainfield butterfly loops of short-tailed BTA-C6 and C8 devices, the corresponding electromechanical response of other BTA homologues (C10, C12, C18) was mostly caused by the Maxwell strain in combination with a pronounced softening of the bulk upon polarization switching. The small-signal response remained normal, i.e. negative, with gradually decreasing $d_{33}$ values for longer substituted molecules.

Based on these results, BTA is the first small-molecular organic ferroelectric with an experimentally observed negative piezoelectric effect. Combined with low fatigue $\left(>10^{6}\right.$ cycles for full polarization switching), long polarization retention ( $>10$ years at room temperature) and a broad operating temperature range, the disorder-tuned piezoelectric response with $d_{33}$ potentially over $-20 \mathrm{pm} \mathrm{V}^{-1}$ and a large figure of merit for energy harvesting $d_{33} g_{33}=2 \times 10^{-12} \mathrm{~m}^{3} \mathrm{~J}^{-1}$ makes BTA a perspective material for flexible and lightweight piezoelectric applications.

\section{Methods}

\section{Materials and devices}

BTA-C6, C8, C10, C12 and C18 (corresponding to linear alkyl chains $\mathrm{C}_{n} \mathrm{H}_{n+1}$ ) were synthesized following the procedure reported previously. ${ }^{29,31} \mathrm{P}(\mathrm{VDF}-\mathrm{TrFE})$ 77\%/23\% was bought from Solvay and used as received. Thin-film capacitor devices were fabricated from solution by spin-coating on chemically cleaned glass substrates with patterned metal electrodes. $\mathrm{P}(\mathrm{VDF}-\mathrm{TrFE})$ layers were additionally annealed at $140{ }^{\circ} \mathrm{C}$ for 2 hours to increase crystallinity. Top electrodes were thermally evaporated to form cross-bar geometry capacitor devices. Aluminum electrodes were used for BTAs and gold for P(VDF-TrFE). Ferroelectric layers were poled by continuous voltage sweeping prior to further measurements. More Experimental details can be found in the ESI. $\dagger$

\section{Converse piezoelectric effect measurements}

Strain-field piezoelectric characteristics were measured using the commercial vibration-damped AixAcct double-beam laser 
interferometer (DBLI) system with resolution of $10^{-2} \AA$ and $d_{33}$ measurement accuracy of $0.2 \mathrm{pm} \mathrm{V}^{-1}$. Large-signal piezoelectric response was measured during a voltage sweep of $10-50 \mathrm{~Hz}$ and averaged 50-100 times to avoid secondary effects. Polarization (displacement) hysteresis loops were registered simultaneously. Remnant strain was set to zero. Small-signal piezoelectric response and small-signal capacitance were measured by applying a staircase-like voltage signal with superimposed high-frequency sinusoidal voltage signal $\left(f_{\mathrm{ss}}=1-5 \mathrm{kHz}, V_{\mathrm{ss}}=\right.$ 1-5 V). As the ferroelectric layer is clamped on a rigid substrate, all piezoelectric characteristics obtained should be considered effective. Measurement temperature was controlled by Linkam heating stage system.

\section{Author contributions}

I. U. performed experiments, analysed the data and wrote the manuscript draft. X. M. and S. B. synthesized and characterized the BTA materials. Y. W. helped with the piezoelectric measurements. M. B. and M. L. executed the DFT/MD simulations. I. U., T. D. C. and M. K. wrote and edited the paper. M. K. and M. L. supervised the work. All authors discussed the results and reviewed the paper.

\section{Data and materials availability}

Data and materials are available from the corresponding author upon reasonable request.

\section{Conflicts of interest}

Authors declare no competing interests.

\section{Acknowledgements}

We would like to thank Prof. Beatriz Noheda Pinuaga for the opportunity to use the piezoelectric response measurement equipment and for stimulating discussions. We also thank Jacob Baas for the technical support during the piezoelectric measurements. We gratefully acknowledge Rint Sijbesma for guiding the materials synthesis and feedback on the manuscript. I. U. acknowledges funding by Vetenskapsrådet. Y. W. thanks the China Scholarship Council for funding. T. D. C. acknowledges financial support from the Swedish Government Strategic Research Area in Materials Science on Functional Materials at Linköping University (Faculty Grant SFO Mat LiU No. 2009 00971). M. L. thanks the Swedish e-Science Research Center (SERC) for funding and the Swedish National Infrastructure for Computing (SNIC) for providing computer resources.

\section{References}

1 C. Brown, R. Kell, R. Taylor and L. Thomas, Piezoelectric Materials, A Review of Progress, IRE Trans. Compon. Parts, 1962, 9, 193-211.
2 W. L. Bond, A Mineral Survey for Piezo-Electric Materials, Bell Syst. Tech. J., 1943, 22, 145-152.

3 P. K. Panda, Review: environmental friendly lead-free piezoelectric materials, J. Mater. Sci., 2009, 44, 5049-5062.

4 K. S. Ramadan, D. Sameoto and S. Evoy, A review of piezoelectric polymers as functional materials for electromechanical transducers, Smart Mater. Struct., 2014, 23, 33001.

5 S. B. Lang and S. Muensit, Review of some lesser-known applications of piezoelectric and pyroelectric polymers, Appl. Phys. A: Mater. Sci. Process., 2006, 85, 125-134.

6 J. Rödel, et al., Transferring lead-free piezoelectric ceramics into application, J. Eur. Ceram. Soc., 2015, 35, 1659-1681.

7 A. H. Rajabi, M. Jaffe and T. L. Arinzeh, Piezoelectric materials for tissue regeneration: A review, Acta Biomater., 2015, 24, 12-23.

8 D.-W. Fu, et al., Diisopropylammonium Bromide Is a HighTemperature Molecular Ferroelectric Crystal, Science, 2013, 339, 425-428.

9 S. Horiuchi, et al., Above-room-temperature ferroelectricity in a single-component molecular crystal, Nature, 2010, 463, 789-792.

10 K. Kobayashi, S. Horiuchi, S. Ishibashi, Y. Murakami and R. Kumai, Field-Induced Antipolar-Polar Structural Transformation and Giant Electrostriction in Organic Crystal, J. Am. Chem. Soc., 2018, 140, 3842-3845.

$11 \mathrm{~W}$. Gao, et al., Flexible organic ferroelectric films with a large piezoelectric response, NPG Asia Mater., 2015, 7, e189.

12 Z. Zhang, et al., Tunable electroresistance and electro-optic effects of transparent molecular ferroelectrics, Sci. Adv., 2017, 3, e1701008.

13 T. Furukawa and N. Seo, Electrostriction as the Origin of Piezoelectricity in Ferroelectric Polymers, Jpn. J. Appl. Phys., 1990, 29, 675-680.

14 I. Katsouras, et al., The negative piezoelectric effect of the ferroelectric polymer poly(vinylidene fluoride), Nat. Mater., 2016, 15, 78-84.

15 Y. Liu, et al., Ferroelectric polymers exhibiting behaviour reminiscent of a morphotropic phase boundary, Nature, 2018, 562, 96-100.

16 M. Owczarek, et al., Flexible ferroelectric organic crystals, Nat. Commun., 2016, 7, 13108.

17 Y.-M. You, et al., An organic-inorganic perovskite ferroelectric with large piezoelectric response, Science, 2017, 357, 306-309.

18 S. Horiuchi, J. Tsutsumi, K. Kobayashi, R. Kumai and S. Ishibashi, Piezoelectricity of strongly polarized ferroelectrics in prototropic organic crystals, J. Mater. Chem. C, 2018, 6, 4714-4719.

19 D. B. Deutz, et al., Analysis and experimental validation of the figure of merit for piezoelectric energy harvesters, Mater. Horiz., 2018, 5, 444-453.

20 R. K. Vasudevan, N. Balke, P. Maksymovych, S. Jesse and S. V. Kalinin, Ferroelectric or non-ferroelectric: Why so many materials exhibit 'ferroelectricity' on the nanoscale, Appl. Phys. Rev., 2017, 4, 21302.

21 B. Noheda, Structure and high-piezoelectricity in lead oxide solid solutions, Curr. Opin. Solid State Mater. Sci., 2002, 6, 27-34. 
22 S. Liu and R. E. Cohen, Origin of Negative Longitudinal Piezoelectric Effect, Phys. Rev. Lett., 2017, 119, 207601.

23 H. S. Nalwa, Ferroelectric Polymers: Chemistry, Physics, and Applications, CRC Press, Boca Raton, 1995.

24 B. Ploss, B. Ploss, F. G. Shin, H. L. W. Chan and C. L. Choy, Pyroelectric or piezoelectric compensated ferroelectric composites, 2000.

25 I. Graz, et al., Flexible active-matrix cells with selectively poled bifunctional polymer-ceramic nanocomposite for pressure and temperature sensing skin, J. Appl. Phys., 2009, 106, 34503.

26 E. Fukada, History and recent progress in piezoelectric polymers, IEEE Trans. Ultrason. Ferroelectr. Freq. Control, 2000, 47, 1277-1290.

27 T. Lenz, et al., Ferroelectricity and piezoelectricity in soft biological tissue: Porcine aortic walls revisited, Appl. Phys. Lett., 2017, 111, 133701.

28 C. F. C. Fitié, W. S. C. Roelofs, M. Kemerink and R. P. Sijbesma, Remnant polarization in thin films from a columnar liquid crystal, J. Am. Chem. Soc., 2010, 132, 6892-6893.

29 C. F. C. Fitié, et al., Polar Switching in Trialkylbenzene-1,3,5tricarboxamides, J. Phys. Chem. B, 2012, 116, 3928-3937.

30 A. V. Gorbunov, et al., True ferroelectric switching in thin films of trialkylbenzene-1,3,5-tricarboxamide (BTA), Phys. Chem. Chem. Phys., 2016, 18, 23663-23672.

31 I. Urbanaviciute, et al., Tuning the Ferroelectric Properties of Trialkylbenzene-1,3,5-tricarboxamide (BTA), Adv. Electron. Mater., 2017, 3, 1600530.

32 A. V. Gorbunov, et al., Polarization loss in the organic ferroelectric trialkylbenzene-1,3,5-tricarboxamide (BTA), Phys. Chem. Chem. Phys., 2017, 19, 3192-3200.

33 I. Urbanaviciute, et al., Suppressing depolarization by tail substitution in an organic supramolecular ferroelectric, Phys. Chem. Chem. Phys., 2019, 21, 2069-2079.

34 A. V. Gorbunov, et al., Switchable Charge Injection Barrier in an Organic Supramolecular Semiconductor, ACS Appl. Mater. Interfaces, 2016, 8, 15535-15542.
35 A. V. Gorbunov, et al., Ferroelectric self-assembled molecular materials showing both rectifying and switchable conductivity, Sci. Adv., 2017, 3, e1701017.

36 W. Y. Pan and L. E. Cross, A sensitive double beam laser interferometer for studying high-frequency piezoelectric and electrostrictive strains, Rev. Sci. Instrum., 1989, 60, 2701-2705.

37 P. Gerber, C. Kügeler, U. Böttger and R. Waser, Effects of reversible and irreversible ferroelectric switchings on the piezoelectric large-signal response of lead zirconate titanate thin films, J. Appl. Phys., 2005, 98, 124101.

38 D. Kluge, et al., Influence of the Molecular Structure and Morphology of Self-Assembled 1,3,5-Benzenetrisamide Nanofibers on their Mechanical Properties, Small, 2012, 8, 2563-2570.

39 T. Furukawa, Piezoelectricity and pyroelectricity in polymers, IEEE Trans. Electr. Insul., 1989, 24, 375-394.

40 F. Preisach, Über die magnetische Nachwirkung, Z. Phys., 1935, 94, 277-302.

41 I. Urbanavičiūtè, T. D. Cornelissen, X. Meng, R. P. Sijbesma and M. Kemerink, Physical reality of the Preisach model for organic ferroelectrics, Nat. Commun., 2018, 9, 4409.

42 V. Khikhlovskyi, et al., Multi-bit organic ferroelectric memory, Org. Electron., 2013, 14, 3399-3405.

43 A. V. Gorbunov, et al., Polarization loss in the organic ferroelectric trialkylbenzene-1,3,5-tricarboxamide (BTA), Phys. Chem. Chem. Phys., 2017, 19, 3192-3200.

44 R. Pelrine, R. Kornbluh, Q. Pei and J. Joseph, High-speed electrically actuated elastomers with strain greater than 100\%, Science, 2000, 287, 836-839.

45 A. O'Halloran, F. O'Malley and P. McHugh, A review on dielectric elastomer actuators, technology, applications, and challenges, J. Appl. Phys., 2008, 104, 71101.

46 S.-W. Hahm and D.-Y. Khang, Crystallization and microstructuredependent elastic moduli of ferroelectric $\mathrm{P}(\mathrm{VDF}-\mathrm{TrFE})$ thin films, Soft Matter, 2010, 6, 5802.

47 C. S. Wojnar, J.-B. le Graverend and D. M. Kochmann, Broadband control of the viscoelasticity of ferroelectrics via domain switching, Appl. Phys. Lett., 2014, 105, 162912. 